\title{
A simplified system for simulation of Streptococcus mutans biofilm on healthy extracted human tooth as dental plaque model
}

\author{
Nadia Halib*, Nor Zaihana Abdul Rahman, Rohazila Mohamad Hanafiah, Nabihah Roslan, Nurhani Jauhar \\ Faculty of Dentistry, Universiti Sains Islam Malaysia (USIM), Kuala Lumpur, Malaysia.
}

\begin{tabular}{l}
\hline ARTICLE INFO \\
\hline Received on: $24 / 09 / 2018$ \\
Accepted on: $14 / 12 / 2018$ \\
Available online: $28 / 02 / 2019$ \\
\\
\hline Key words: \\
In vitro biofilm, plaque \\
simulation, extracted tooth, \\
Streptococcus mutans.
\end{tabular}

\begin{abstract}
The understanding and capability to simulate reproducible in vitro plaque model are important where this artificial plaque can be utilized as a tool in assessing the efficacy of tooth cleaning products. Previous studies were done on synthetic surfaces such as glass beads under a very strict, controlled environment. Thus, the aim of this study was to establish a simplified system to enable the formation of single species biofilm on the extracted natural tooth as plaque model. Healthy extracted natural tooth was incubated in broth mixture of Streptococcus mutans, containing $5 \%$ sucrose and stimulated saliva in a simplified system. The incubation was set at room temperature for 24,48 , and 72 hours. After 24 hours, the sticky deposit was formed on the surface of the tooth. The area of biofilm, stained with disclosing agent, expended from $19.05 \pm 1.41 \mathrm{~mm}^{2}$ (24 hours) to $23.25 \pm 0.35 \mathrm{~mm}^{2}$ (48 hours). However, no significant expansion of the plaque area was observed on 72 hours $\left(23.83 \pm 0.52 \mathrm{~mm}^{2}\right)$. The biofilm was then observed under Infinite Focus Microscope (IFM) to measure the biofilm thickness. The preliminary attempt showed that plaque appeared as dark deposit with a certain thickness. Therefore, this simplified system is a reliable method for producing artificial plaque.
\end{abstract}

\section{INTRODUCTION}

Human oral cavity is a complex ecological environment where microorganisms are bathed in a liquid medium such as saliva. These microorganisms have access and adhere to both hard and soft tissue surfaces; hence, facilitate the development of biofilms. Dental plaque is the film of micro-organisms found on the tooth surface embedded in a matrix of polymers of salivary and bacterial origin (Marsh, 1992). It has a heterogenous, biodiverse but organized structure that changes over time (Listgarten, 1994). The development and maturation of dental plaque as a biofilm has profound implication in the etiology and progression of dental caries and periodontal diseases (Filoche et al., 2010).

The understanding towards dental plaque formation is important in the development of an effective cleaning aid in order to help reduce or slowing down the formation process. However, in vivo experimental studies of natural dental plaque in both humans

"Corresponding Author

Nadia Halib, Faculty of Dentistry, Universiti Sains Islam Malaysia (USIM), Kuala Lumpur, Malaysia.E-mail:nadia.halib@usim.edu.my and animals are severely restricted and involved complicated ethical issues, such as induction of gingivitis by simple plaque accumulation and problems with access and sampling (Sissons, 1997). Therefore, in vitro model systems which are more controllable have been recently developed and used to explain and predict its behavior. In those studies, the biofilm was simulated as artificial dental plaque onto surfaces of various materials such as hydroxyapatite beads, glass beads, orthodontic wire, and inert surface like Teflon in the constant depth film fermenter (Appelbaum et al., 1979; Jacob, 2006; Matsumoto et al., 2004; WI et al., 2013). While others simulate dental plaque in situ using bovine dentin disks worn by human subjects (Zaura-Arite et al., 2001).

A more complex model was also developed to mimic the constant saliva or gingival crevicular fluid flow conditions. Blanc et al. (2014) had used bioreactor to grow the oral bacteria under flow and shear conditions and found that their system was highly reproducible for multispecies oral biofilm formation. The system had become a useful tool for evaluating the activity of three antiplaque agents (Blanc et al., 2014).

The simulation of artificial dental plaque on extracted natural tooth was first done, by Jordan and Keyes (1966) which 
utilized streptococci isolated from rats and hamster to form the biofilm. In our study, we aimed to establish a simplified system of single species, manipulating Streptococcus mutans to form stable and reproducible biofilm onto healthy extracted human tooth which later used as a model to evaluate the efficacy of our in-house production of tooth cleaning aid. We expected that the formation of biofilm was possible in a very basic condition with minimum control over the set environment as in our simplified system.

\section{MATERIALS AND METHODS}

\section{Tooth preparation}

In this study, no patient or human volunteer was involved. The extracted teeth were provided by Orthodontics Clinic, Faculty of Dentistry, Universiti Sains Islam Malaysia (USIM). The teeth were indicated for extraction because of crowding in patient required to wear braces. However, the procedure of handling human samples in this study had been approved by the Faculty

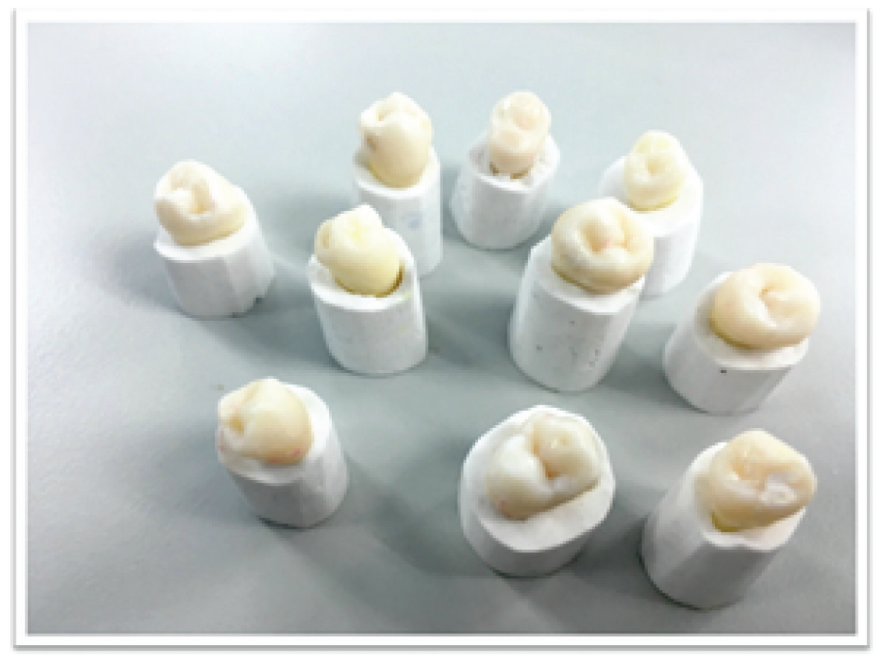

Figure 1. Teeth mounted in gypsum covering from the apex of the root to the cementoenamel line. of Dentistry, USIM ethical committee. Healthy extracted natural premolar teeth free from plaque, calculus, caries, and restoration were selected. Teeth were then mounted in gypsum, covering from the apex of the root to the cementoenamel line, as shown in Figure 1.

\section{Bacterial strains}

Streptococcus mutans (ATCC25175) was grown on nutrient agar at $\mathrm{pH} 7$ and incubated at $37^{\circ} \mathrm{C}$ under anaerobic condition $\left(10 \% \mathrm{H}_{2}, 10 \% \mathrm{CO}_{2}\right.$, and $\left.80 \% \mathrm{~N}_{2}\right)$. The bacterial colony was then inoculated into $10 \mathrm{ml}$ of Brain Heart Infusion broth (source) and incubated at $37^{\circ} \mathrm{C}$ for 72 hours. The bacterial concentration was measured using spectrophotometer at $540 \mathrm{~nm}$.

\section{Media}

Sucrose $5 \%$ was used for plaque simulation. To make $5 \%$ sucrose, $0.5 \mathrm{ml}$ of $100 \%$ sucrose stock was added into $10 \mathrm{ml}$ brain heart infusion (BHI) broth containing Streprococcus mutans (BHI-Sm).

\section{Saliva collection}

Saliva samples were collected from one healthy volunteer using the spit-out method after chewing $1 \mathrm{~g}$ of paraffin wax for 5 minutes (Navazesh, 1993). The collection of saliva was performed 1 hour after breakfast between 09:30 and 11:30 in the morning. All experimental and saliva collection protocols were approved by the Faculty of Dentistry, USIM ethical committee.

\section{Biofilm simulation procedure}

The mounted tooth was first placed in a universal bottle containing a mixture of $10 \mathrm{ml}$ BHI broth containing Streptococcus mutans at $10^{8} \mathrm{CFU} / \mathrm{ml}$ and $0.5 \mathrm{ml}$ of $100 \%$ sucrose. Another universal bottle was filled with $3 \mathrm{ml}$ of saliva. Both universal bottles (containing saliva and BHI-S. mutan media) were connected to each other with $5 \mathrm{~mm}$ silicon tube. The middle part of the tube was attached to a peristaltic pump to create circulation and placed in arrangement set-up, as shown in Figure 2. The pump

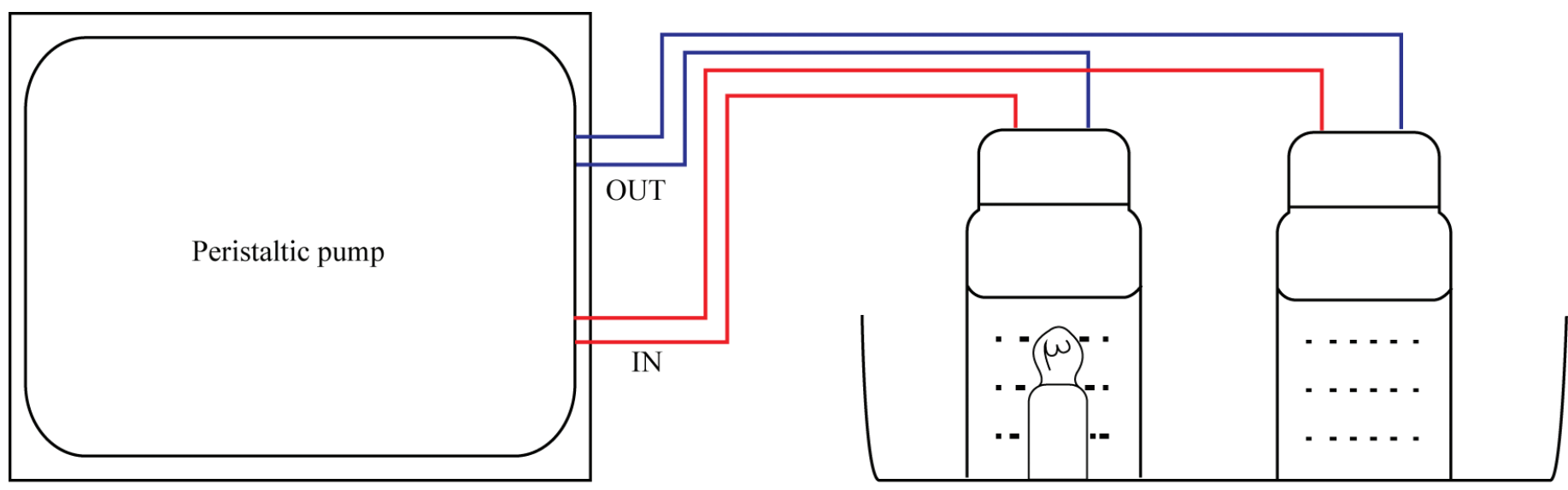

Brain heart infusion broth (BHI)

Saliva containing Streprococcus mutans

Figure 2. Set-up for a simplified plaque simulation system. 
was set at $32 \mathrm{rpm}$ and circulation was continuously allowed at room temperature for 24,48 , and 72 hours.

To evaluate the biofilm formation, the mounted tooth was rinsed and later stained using erythrosine as the staining agent. The tooth was soaked in the dye and again rinsed off to remove the excessive stain with normal saline before the area covered with biofilm was measured. The thickness of the plaque was analyzed using infinite focus microscope (IFM).

\section{RESULT AND DISCUSSION}

After 24 hours of incubation, the sticky deposit was observed on the tooth surface. The sticky deposit appeared red when stained with erythrosine; hence, confirms the presence of dental biofilm (Fig. 3). The area covered with a plaque was measured three times and recorded in mean value (Table 1). The measurement of biofilm formation was repeated again after 48- and 72-hours incubation. It was found that the biofilm area was $19.05 \pm 1.41 \mathrm{~mm}^{2}$ for 24 hours of incubations. And the area expended to $23.25 \pm 0.35 \mathrm{~mm}^{2}$ after 48 hours of incubation.

In this experiment, it was observed that our simplified system was capable of producing biofilm onto the surface of the healthy extracted tooth. The $5 \%$ sucrose in our system also granted the expansion of biofilm formation up to 48 hours. However, further incubation to 72 hours did not exhibit significant progress of growth $\left(23.83 \pm 0.52 \mathrm{~mm}^{2}\right)$ which suggested that extension of incubation time requires additional of sucrose source.

The replacement of saliva with buffer in this system did not favor the formation of biofilm. This is because saliva is required for the formation of an acellular organic pellicle on the exposed tooth surface (Rosan and Lamont, 2000). This organic coating (salivary pellicle) is derived from adsorption of mucinous proteins from the saliva (Hicks et al., 2004). This finding was in agreement with the previous study where Jordan and Keyes (1966) observed if one of two factors (saliva and sucrose) was replaced, no visible biofilm was formed. They found that synthetic saliva was required for plaque production
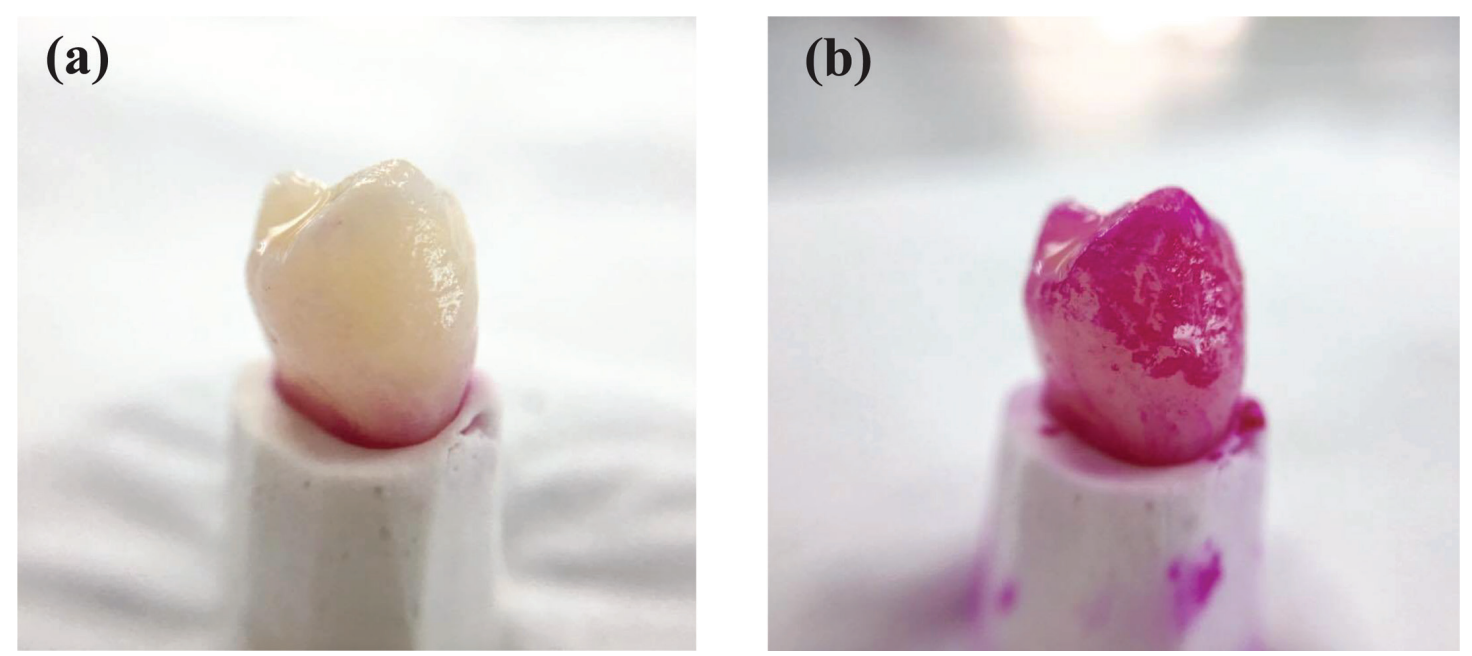

Figure 3. Photographs showing biofilm formation on the natural extracted tooth (a) before and (b) after erythrosine staining. but the various salt components were not and glucose, fructose, galactose, sorbitol, lactose, or a mixture of glucose and fructose could not be substituted for sucrose (Jordan and Keyes, 1966).

The formation of biofilm was found to be reproducible in this system. Although it has basic control of the environment (fixed parameters in temperature and amount of glucose and bacteria concentration), the influence of external factors was still within the limit where variables have less influence in the formation of biofilm. This will aid in acquiring reliable data in the evaluation of tooth cleaning product efficacy (for example, the mechanical cleansing agent such as toothbrushing).

The biofilm was later observed under IFM. The initial idea was to evaluate the topology of the biofilm and subsequently estimated the thickness of biofilm formation. However, the first attempt only allowed us to visualize plaque as a dark deposit, as shown in Figure 4. The thickness cannot be properly measured as the plaque was dried out during the procedure. Thus, a proper method of sample preparation is required to be established in the near future.

\section{CONCLUSION}

In this study, the simplified system was able to simulate a reproducible artificial plaque. The system managed to produce artificial dental plaque by the formation of biofilm on the surface of the natural tooth. The area of biofilm covering the tooth surface expended as the incubation time increased from 24 to 48 hours. This system is easy to set and use as it only requires simple apparatus and simulation run at room temperature. The artificial plaque produced in this simplified

Table 1. Area of biofilm formation on the tooth surface.

\begin{tabular}{lc}
\hline & Area of biofilm on the tooth surface $\left(\mathbf{m m}^{2}\right)$ \\
\hline 24 hours & $19.05 \pm 1.41$ \\
48 hours & $23.25 \pm 0.35$ \\
72 hours & $23.83 \pm 0.52$ \\
\hline
\end{tabular}




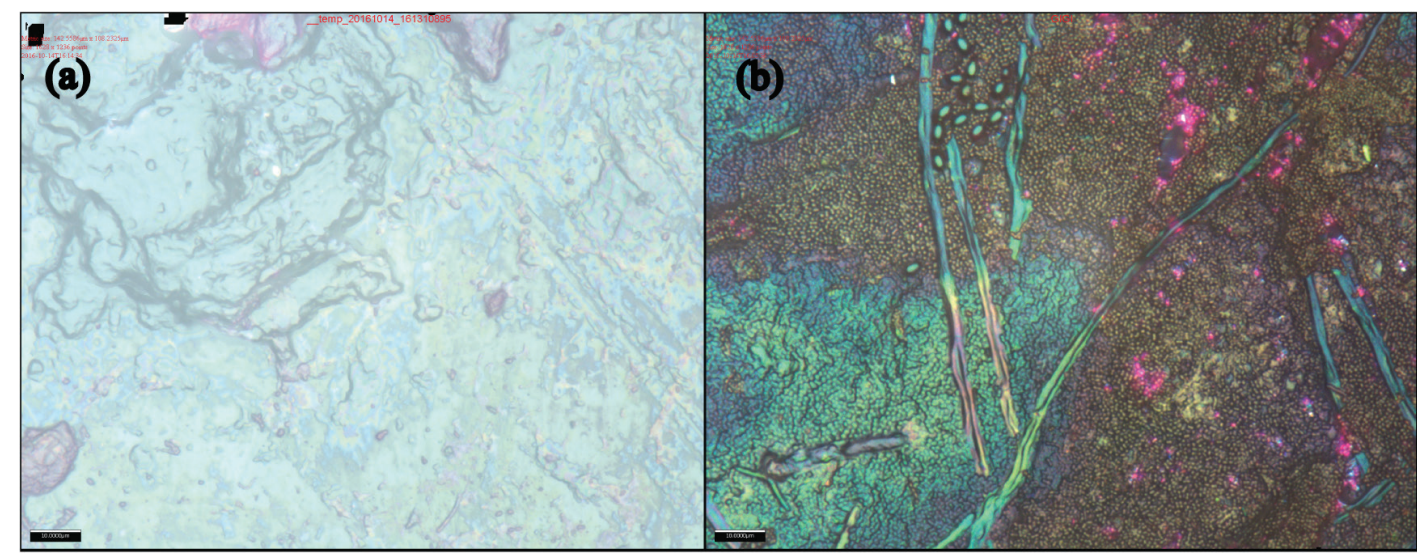

Figure 4. IFM micrographs showing (a) clean tooth surface, (b) biofilm formation on the extracted natural tooth surface.

system will benefit as it is further utilized as a tool to evaluate tooth cleaning product efficacy.

\section{ACKNOWLEDGMENTS}

The author would like to acknowledge the Ministry of Higher Education (MOHE), Malaysia for the Fundamental Research Grant Scheme (FRGS/1/2014/SG06/USIM/02/1) and Universiti Sains Islam Malaysia Research Grant Scheme (PPP/ USG-0216/FPG/30/16416) that has funded this study.

\section{REFERENCES}

Appelbaum B, Golub E, Holt SC, Rosan B. In vitro studies of dental plaque formation: adsorption of oral streptococci to hydroxyaptite. Infect Immun, 1979; 25:717-28

Blanc V, Isabal S, Sanchez MC, Llama-Palacios A, Herrera D, Sanz M, León R. Characterization and application of a flow system for in vitro multispecies oral biofilm formation. J Periodontal Res, 2014; 49(3):323-32.

Filoche S, Wong L, Sissons CH. Oral biofilms: emerging concepts in microbial ecology. J Dent Res, 2010; 89:8-18.

Hicks J, Garcia-Godoy F, Flaitz C. Biological factors in dental caries: role of saliva and dental plaque in the dynamic process of demineralization and remineralization (part 1). J Clin Pediatr Dent, 2004; $28: 47-52$

Jacob M. Biofilms, a new approach to the microbiology of dental plaque. Odontology, 2006; 94:1-9.

Jordan H, Keyes P. In vitro methods for the study of plaque formation and carious lesions. Arch Oral Biol, 1966; 11:793-801.
Listgarten MA. The structure of dental plaque. Periodontology, 2000, 1994; 5:52-65.

Marsh P. Microbiological aspects of the chemical control of plaque and gingivitis. J Dent Res, 1992; 71:1431-8.

Matsumoto M, Tsuji M, Okuda J, Sasaki H, Nakano K, Osawa $\mathrm{K}$, Shimura S, Ooshima T. Inhibitory effects of cacao bean husk extract on plaque formation in vitro and in vivo. Eur J Oral Sci, 2004; 112:249-52.

Navazesh M. Methods for collecting saliva. Ann NY Acad Sci, $1993 ; 694: 72-7$.

Rosan B, Lamont RJ. Dental plaque formation. Microbes Infect, 2000; 2:1599-607.

Sissons C. Artificial dental plaque biofilm model systems. Adv Dental Res, 1997; 11:110-26.

WI WNH, Fathilah AR, Rahim ZH. Plant extracts of Psidium guajava, Mangifera and Mentha sp. inhibit the growth of the population of single-species oral biofilm. Altern Integr Med, 2013; 2(1):1-6.

Zaura-Arite E, Van Marle J, Ten Cate JM. Confocal microscopy study of undisturbed and chlorhexidine-treated dental biofilm. J Dent Res, $2001 ; 80: 1436-40$

\section{How to cite this article:}

Halib N, Rahman NZA, Hanafiah RM, Roslan N, Jauhar N. A simplified system for simulation of Streptococcus mutans biofilm on healthy extracted human tooth as dental plaque model. J Appl Pharm Sci, 2019; 9(02):112-115. 